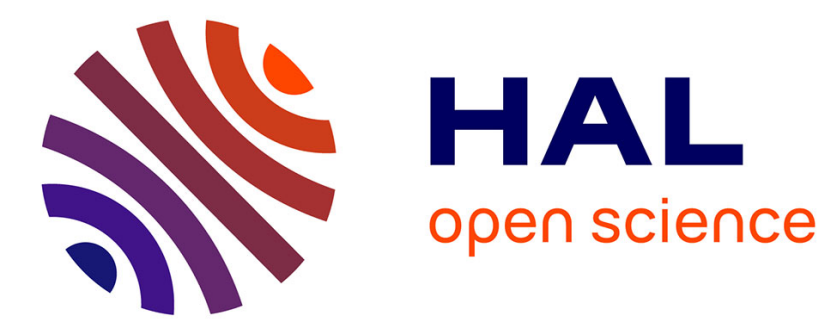

\title{
THE ADDITIONAL DETERMINATION OF THE PHASE DIFFERENCE IN REFLECTION SPECTROSCOPY
}

W. Plieth

\section{- To cite this version:}

W. Plieth. THE ADDITIONAL DETERMINATION OF THE PHASE DIFFERENCE IN REFLECTION SPECTROSCOPY. Journal de Physique Colloques, 1977, 38 (C5), pp.C5-215-C5-224. 10.1051/jphyscol:1977528 . jpa-00217177

\section{HAL Id: jpa-00217177 https://hal.science/jpa-00217177}

Submitted on 1 Jan 1977

HAL is a multi-disciplinary open access archive for the deposit and dissemination of scientific research documents, whether they are published or not. The documents may come from teaching and research institutions in France or abroad, or from public or private research centers.
L'archive ouverte pluridisciplinaire HAL, est destinée au dépôt et à la diffusion de documents scientifiques de niveau recherche, publiés ou non, émanant des établissements d'enseignement et de recherche français ou étrangers, des laboratoires publics ou privés. 


\title{
THE ADDITIONAL DETERMINATION OF THE PHASE DIFFERENCE IN REFLECTION SPECTROSCOPY
}

\author{
W. J. PLIETH \\ Institut für Physikalische Chemie der Freien Universität Berlin \\ 1 Berlin 33, Fabeckstrasse 32, Germany
}

Résumé. - Des phases intermédiaires, ayant une anisotropie uniaxiale, sont caractérisées par les parties réelles et imaginaires des intégrales

$$
\int_{1}^{3}\left(\hat{\varepsilon_{t}}-\hat{\varepsilon}_{\mathrm{ret}}\right) \mathrm{d} z, \quad \int_{1}^{3}\left(\frac{1}{\hat{\varepsilon}_{\mathrm{n}}}-\frac{1}{\widehat{\varepsilon}_{\mathrm{ret}}}\right) \mathrm{d} z .
$$

Ces résultats sont obtenus par application stricte de la théorie de continuum de Maxwell pour le cas d'une frontière de phase perturbée utilisant des conditions intégrales de limite. Pour la détermination complète de ces quantités, quatre valeurs expérimentales sont nécessaires. Celles-ci sont les réflectivités $\Delta R / R$ et les différences de phase $\Delta \varphi$ qu'on peut obtenir à l'aide de la spectroscopie de réflexion avec de la lumière polarisée parallèlement et perpendiculairement. La méthode mathématique des transformations de type « Kramers-Kronig » et la détermination expérimentale de $\Delta \varphi$ par interférométrie sont décrites. L'application de la méthode "Kramers-Kronig » est démontrée pour le cas des couches d'oxyde de platine sur Pt et pour l'adsorption de rhodamine B sur Pt et Ag. Pour le cas du système oxyde de platine sur Pt la componente tangentielle $\widehat{\varepsilon}_{\mathrm{t}}$ et la componente normale $\widehat{\varepsilon}_{n}$ de $\widehat{\varepsilon}$ sont déterminées en plus. Dans le cas de couches organiques adsorbées, les intégrales sont discutćes en tenant compte de l'orientation des molécules organiques sur la surface.

\begin{abstract}
It follows from a dcrivation of Fresnel's reflection coefficients using integral boundary conditions that within the framework of the Maxwell continuum theory four quantities can be determined for an interface with uniaxial anisotropy. These quantities are the real and imaginary parts of the integrals

$$
\int_{1}^{3}\left(\hat{\varepsilon_{\mathrm{t}}}-\widehat{\varepsilon_{\mathrm{ret}}}\right) \mathrm{d} z, \quad \int_{1}^{3}\left(\frac{1}{\hat{\varepsilon}_{\mathrm{n}}}-\frac{1}{\hat{\varepsilon}_{\mathrm{ret}}}\right) \mathrm{d} z .
$$

$\left(\hat{\varepsilon_{t}}=\right.$ tangential component, $\hat{\varepsilon}_{\mathrm{n}}$ normal component of the dielectric constant tensor, $\hat{\varepsilon}_{\mathrm{ret}}$ dielectric constant of the reference state). To determine these quantities four experimental values are necessary. These values can be obtained by the additional determination of the phase change $\Delta \varphi$ in reflection spectroscopy with parallel and perpendicular polarized light. The Kramers-Kronig transformation method and the possible measurement of $\Delta \varphi$ by interferometry are described. Examples for the Kramers-Kronig transformation method are the determination of the phase change during reflection $\Delta \varphi$ for platinum oxide layers on $\mathrm{Pt}$ and for rhodamin $\mathrm{B}$ adsorption layers on $\mathrm{Pt}$ and $\mathrm{Ag}$. From $\Delta R / R$ and $\Delta \varphi$ the integrals are calculated. In the case of platinum oxide on Pt additionally the tangential and normal component of $\hat{\varepsilon}$ are derived. In the case of the organic adsorption layers the integrals are discussed with regard to the orientation of the organic molecules on the surface.
\end{abstract}

1. Introduction. - In some of the first papers on spectroscopic methods for studying solid-liquid interfaces the determination of optical constants of surface layers was given main priority. Lateron the value of these data was questioned. Therefore a critical discussion of the problems encountered is appropriate.

The application of Maxwell's theory for the description of the optical properties of thin surface films down to monomolecular dimensions has been subject to several objections. This means a critical discussion of optical constants of such films. Also anisotropic differences in the optical properties have to be taken into account. Wrong values are calculated if the anisotropy is neglected. But to allow for anisotropy an extension of the experimental parameters is necessary. After a discussion of the first points the determination of the phase shift is described as the additional parameter in reflection spectroscopy to deal with anisotropy.

2. The equations for the Fresnel reflection coefficient of interfaces with thin surface layers. - In figure 1, curve $a$, the absolute value of a complex dielectric constant $|\hat{\varepsilon}|$ across a phase boundary is shown as a function of the coordinate $z$ normal to the surface. The value of $|\hat{\varepsilon}|$ for a differential surface element varies discontinuously from atom to atom. But averaging the $\hat{\varepsilon}$-values for all the surface elements $|\widehat{\varepsilon}|$ becomes a continuous function of $z$. The reason for this conti- 
nuous behaviour are the different locations of the surface atoms on the $z$-axis for different surface elements. For smooth surfaces this is caused by vibrations and motions of the surface atoms. For rough surfaces the averaging effect causes a large extension of the surface area into the adjacent phases.

With one or more surface layers or surface films present on the surface $|\widehat{\varepsilon}|$ may become a rather complicated function of $z$ but the average value over the surface elements maintains its continuous behaviour (Fig. 1, curve b). The layers and films we think

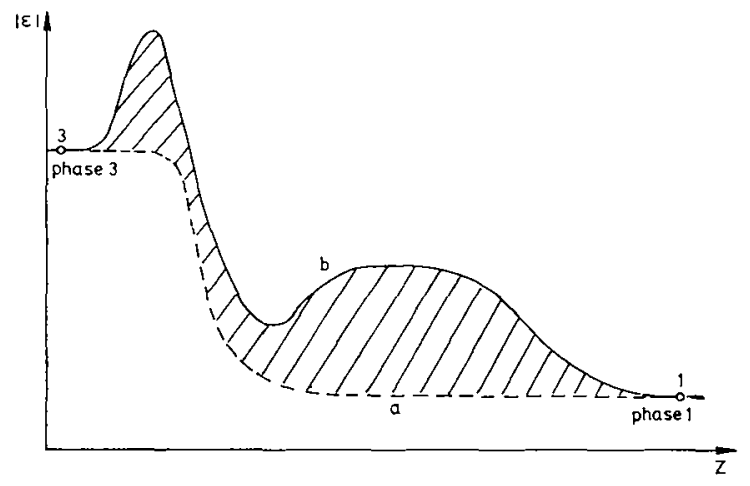

Frg. 1. - Continuous behaviour of the average value of the complex dielectric constant at an interface; curve $a:$ film free surface (reference state) ; curve $b$ : surface with an electrochemical double layer and specific adsorption. Points 1 and 2 refer to the integration limits in eqs (1) and (2). The hatched area represents the integral $\int\left(\widehat{\varepsilon}-\hat{\varepsilon}_{\mathrm{ref}}\right) \mathrm{d} z$ in eq. (2).

of are electric double layers, adsorption layers or films of only a few monolayers. Thus figure 1 , curve $b$, shows the situation of a metal-electrolyte boundary with a charge layer in the metal and an adsorption layer on the side of the electrolyte.

So far we considered an isotropic value of $|\hat{\varepsilon}|$ In general, it is unlikely that the variation of $|\hat{\varepsilon}|$ across the interface is the same for the different tensor components of the dielectric constant. To take into account these differences one can apply the simplest anisotropic model for the surface area, the case of a uniaxial anisotropic surface region with the optical axis normal to the surface. Then different $|\hat{\varepsilon}|-z$-plots exist for the tensor components of $\hat{\varepsilon}$ tangential $\left(\hat{\varepsilon}_{v}\right)$ and normal $\left(\hat{\varepsilon}_{n}\right)$ to the surface.

It is now possible to derive complex Fresnel reflection coefficients $\hat{r}$ using Maxwell's theory with integral boundary conditions. The resulting equations given by Plieth and Naegele [1] and taking into account the anisotropy are of the following form

$$
\begin{aligned}
\frac{\hat{r}_{\perp}}{\hat{r}_{\perp}^{*}}=1 & -i \frac{4 \pi \sqrt{\varepsilon_{1}} \cos \alpha_{1}}{\lambda\left(\varepsilon_{1}-\hat{\varepsilon}_{3}\right)} \times \\
\times & {\left[\int_{1}^{0}\left(\hat{\varepsilon}_{\mathrm{t}}-\varepsilon_{1}\right) \mathrm{d} z+\int_{0}^{3}\left(\hat{\varepsilon_{1}}-\hat{\varepsilon}_{3}\right) \mathrm{d} z\right] }
\end{aligned}
$$

$$
\begin{aligned}
& \frac{\hat{r}_{\|}}{\hat{r}_{\|}^{*}}=1-i \frac{4 \pi \sqrt{\varepsilon_{1}} \cos \alpha_{1}}{\lambda\left(\varepsilon_{1} \cos ^{2} \hat{\alpha}_{3}-\hat{\varepsilon}_{3} \cos ^{2} \alpha_{1}\right)} \\
& \left\{\left[\int_{1}^{0}\left(\hat{\varepsilon}_{t}-\varepsilon_{1}\right) \mathrm{d} z+\int_{0}^{3}\left(\widehat{\varepsilon_{t}}-\widehat{\varepsilon}_{3}\right) \mathrm{d} z\right]+\right. \\
& \left.+\left[\int_{1}^{0}\left(\frac{1}{\hat{\varepsilon}_{\mathrm{n}}}-\frac{1}{\varepsilon_{1}}\right) \mathrm{d} z+\int_{0}^{3}\left(\frac{1}{\hat{\varepsilon}_{\mathrm{n}}}-\frac{1}{\widehat{\varepsilon}_{3}}\right) \mathrm{d} z\right]\right\}
\end{aligned}
$$

( $\alpha$ angle of incidence, $\lambda$ wavelength of light, indices 1 and 3 refer to the adjacent phases, integration boundaries refer to points in phase 1 and 3 ). The reference values $\hat{r}_{\perp}^{*}$ and $\hat{r}_{\|}^{*}$ are the Fresnel coefficients for a surface with an ideal sharp boundary corrected for the phase difference of the incident beam along the distance $d_{1}$ from integration point 1 to this boundary :

$$
\exp \left(\frac{4 \pi}{\lambda} d_{1} n_{1} \cos \alpha_{1}\right)
$$

This correction is necessary to eliminate the incident light beam with respect to the amplitude as well as to the phase.

The ideal reference value cannot be verified experimentally. The reference surface can best be described by a situation shown in figure 1 , curve a. The equations describing the relative Fresnel coefficients with respect to such a reference state are obtained by deviding eq. $(1 a)$ and $(1 b)$ by similar equations for the reference situation. One obtains by linearization

$$
\begin{aligned}
\frac{\hat{r}_{\perp}}{\hat{r}_{1, \mathrm{ref}}}=1 & -i \frac{4 \pi \sqrt{\varepsilon_{1}} \cos \alpha_{1}}{\lambda\left(\varepsilon_{1}-\hat{\varepsilon}_{3}\right)} \int_{1}^{3}\left(\hat{\varepsilon}_{\mathrm{t}}-\hat{\varepsilon}_{\mathrm{ref}}\right) \mathrm{d} z \\
\frac{\hat{r}_{\mathrm{ll}}}{\widehat{r}_{\mathrm{l}, \mathrm{ref}}}=1 & -i \frac{4 \pi \sqrt{\varepsilon_{1}} \cos \alpha_{1}}{\lambda\left(\varepsilon_{1} \cos ^{2} \hat{\alpha}_{3}-\hat{\varepsilon}_{3} \cos ^{2} \alpha_{1}\right)} \times \\
& \times\left[\int_{1}^{3}\left(\hat{\varepsilon}_{\mathrm{t}}-\hat{\varepsilon}_{\mathrm{rcf}}\right) \mathrm{d} z+\int_{1}^{3}\left(\frac{1}{\hat{\varepsilon}_{\mathrm{n}}}-\frac{1}{\hat{\varepsilon}_{\mathrm{ref}}}\right) \mathrm{d} z\right]
\end{aligned}
$$

Determining the ratio $\widehat{r} / \hat{r}_{\text {ref }}$ the quantities which can be calculated without special assumptions of the model of the interface are the integrals in eq. (2) (hatched area in figure 1). Calculations of $\widehat{\varepsilon}_{1}$ and $\widehat{\varepsilon}_{\mathrm{n}}$ need informations with regard to the interface which cannot be obtained from optical measurements and in many cases are subject to tentative guesses. This concerns the thickness which cannot be calculated from eq. (2) as well as the reference value $\hat{\varepsilon}_{\text {ref }}$. In simple cases $\widehat{\varepsilon}_{\text {ref }}$ can set equal to $\hat{\varepsilon}_{3}$ or $\varepsilon_{1}$. In more complicated cases $\hat{\varepsilon}_{\text {ref }}$ has to be devided into one part $\varepsilon_{1}$ of a layer of thickness $d_{1}$ and one part $\hat{\varepsilon}_{3}$ of a layer of thickness $d_{3}$.

3. The Kramers-Kronig method for calculating the phase change in differential reflection measurements. The two complex Fresnel reflection coefficients $\hat{r} / \widehat{r}_{\text {ref }}$ for parallel and perpendicular polarized light repre- 
sent 4 experimental quantities. With ordinary reflection spectroscopy as well as ellipsometry only two values can be determined. Thus we have to think of extensions of these methods. One way is the additional determination of the phase information lost in reflection spectroscopy. Therefore we investigated the mathematical method of Kramers and Kronig for its application to thin surface films [2]. This method goes back to integrals known as Hilbert or Stieltjes transformations [3] between the real part $G^{\prime}(\hat{\omega})$ and the imaginary part $G^{\prime \prime}(\hat{\omega})$ of a complex function $G(\hat{\omega})=G^{\prime}(\hat{\omega})+i . G^{\prime \prime}(\hat{\omega})$, analytic in the upper half of the complex $\widehat{\omega}$-plane and on the real axis of $\hat{\omega}$. These transformation equations have the form

$$
\begin{aligned}
& G^{\prime}(x)=\frac{1}{\pi} \int_{-\infty}^{+\infty} \frac{G^{\prime \prime}(\omega)}{\omega-x} \mathrm{~d} \omega \\
& G^{\prime \prime}(x)=\frac{1}{\pi} f_{-\infty}^{+\infty} \frac{G^{\prime}(\omega)}{\omega-x} \mathrm{~d} \omega
\end{aligned}
$$

$x$ is a fixed $\omega$-value, the bar in the integral denotes the Cauchy principal value.

An example for the function $\hat{G}$ is the complex dielectric constant $\widehat{\varepsilon}(\hat{\omega})=\varepsilon^{\prime}(\hat{\omega})+i . \varepsilon^{\prime \prime}(\hat{\omega})$. The dependence of $\hat{\varepsilon}$ on the complex frequency $\hat{\omega}$ is described in the literature [4] showing that $\hat{\varepsilon}(\hat{\omega})$ has indeed the analytic properties demanded for $\hat{G}(\hat{\omega})$. The transformation equations can be brought into the form

$$
\begin{aligned}
\varepsilon^{\prime}(x)-1 & =\frac{2}{\pi} \int_{0}^{\infty} \frac{\omega \varepsilon^{\prime \prime}(\omega)}{\omega^{2}-x^{2}} \mathrm{~d} \omega \\
\varepsilon^{\prime \prime}(x) & =\frac{2 x}{\pi} \int_{0}^{\infty} \frac{\varepsilon^{\prime}(\omega)}{\omega^{2}-x^{2}} \mathrm{~d} \omega .
\end{aligned}
$$

The application of eq. (3) is also possible for the Fresnel reflection coefficient $r$ written in the logarithmic form

$$
\ln \hat{r}=\frac{1}{2} \ln R+i\left(\varphi_{\mathrm{r}}-\varphi_{\mathrm{i}}\right)
$$

$R=\hat{r} \cdot \hat{r}^{*}$ is the reflectivity and $\varphi_{\mathrm{r}}-\varphi_{\mathrm{i}}$ denotes the phase jump during reflection. The analyticity of $\widehat{G}=\ln \hat{r}$ must be discussed on the basis of the dependence of $\hat{r}$ on $\hat{\varepsilon}$. For the reflection coefficient of a twophase system the function $\hat{G}$ has the form

$$
\begin{aligned}
& \hat{G}_{\perp}(\hat{\omega})=\ln \frac{\sqrt{\varepsilon_{1}} \cos \alpha_{1}-\sqrt{\sqrt{\varepsilon_{3}}} \cos \hat{\alpha_{3}}}{\sqrt{\varepsilon_{1}} \cos \alpha_{1}+\sqrt{\widehat{\varepsilon_{3}}} \cos \hat{\alpha}_{3}} \\
& \hat{G}_{\|}(\hat{\omega})=\ln \frac{\sqrt{\varepsilon_{1}} \cos \hat{\alpha}_{3}-\sqrt{\widehat{\hat{\varepsilon}_{3}}} \cos \alpha_{1}}{\sqrt{\varepsilon_{1}} \cos \hat{\alpha}_{3}+\sqrt{\widehat{\varepsilon_{3}}} \cos \alpha_{1}} .
\end{aligned}
$$

The functions $\ln \hat{r}$ are not analytic because for $\omega \rightarrow \infty$ goes $\varepsilon_{1} \rightarrow \varepsilon_{3} \rightarrow 1$ and $\hat{r} \rightarrow 0$ and hence $\ln \hat{r} \rightarrow \infty$. But it is shown in the literature [11] that the function $(1+x \omega) /\left(1+\omega^{2}\right) \ln \hat{r}$ is analytic with the exception of a singularity at $\omega=i$. The transformation gives

$$
\begin{aligned}
\Delta \varphi(x) & =\frac{x}{\pi} \int_{0}^{\infty} \frac{\ln R(\omega)}{\omega^{2}-x^{2}} \mathrm{~d} \omega \\
\ln R(x) & =\frac{1}{\pi} f_{0}^{\infty} \frac{\omega \Delta \varphi(\omega)}{\omega^{2}-x^{2}} \mathrm{~d} \omega .
\end{aligned}
$$

The singularity can be taken into account by multiplying $\ln \hat{r}$ with the Blaschke product.

Similarly the relative Fresnel reflection coefficients in eq. (1) and (2) can be discussed. The functions $\hat{G}(\hat{\omega})$ are then represented by the logarithm of these equations. Singularities occur at $\widehat{\varepsilon}_{3}\left(\hat{\omega}_{\mathrm{j}}\right)=\varepsilon_{1}\left(\hat{\omega}_{\mathrm{j}}\right)$ and additional for parallel polarization at $\hat{\varepsilon}_{3}\left(\hat{\omega}_{\mathrm{j}}\right)=\varepsilon_{1}\left(\hat{\omega}_{\mathrm{j}}\right) \tan ^{2} \alpha_{1}$. The transformation equations get the form

$$
\begin{gathered}
\Delta \varphi(x)=-\frac{1}{\pi} \int_{0}^{\infty} \frac{\omega \ln R / R_{\mathrm{ref}}(\omega)}{\omega^{2}-x^{2}} \mathrm{~d} \omega \\
\ln \frac{R}{R_{\mathrm{ref}}}(x)=\frac{x}{\omega} \int_{0}^{\infty} \frac{\Delta \varphi(\omega)}{\omega^{2}-x^{2}} \mathrm{~d} \omega .
\end{gathered}
$$

Again the singularities can be taken into account by multiplying the functions $\ln \widehat{r} / \hat{r}_{\text {ref }}$ with the Blaschke product.

This has the form

$$
\widehat{B}(\widehat{\omega})=\prod_{j} \frac{x-\omega_{j}}{x-\omega_{j}^{*}} .
$$

The result is an additional term $\Sigma_{\mathbf{j}}$ arc $\tan x / \omega_{\mathbf{j}}$ in the eq. (8). These terms can be neglected for $x \ll \omega_{\mathbf{j}}$. The test whether or not a Blaschke product has to be considered is the retransformation of the $\Delta \varphi$-spectrum into the experimental $R / R_{\text {ref }}$-values.

4. The tailfit problem and the calculation procedure. - Theoretically the calculation of the phase change in reflection spectroscopy requires the knowledge of the $R / R_{\mathrm{ref}}$-spectrum for all frequencies from $h v=0$ up to $h v \rightarrow \infty$. Experimentally only a limited frequency range is accessibel (typical range for a metal/electrolyte interface is 1-6 eV). Thus one has to make extrapolations in the regions below and above the interval and to discuss the failure from unproper extrapolations. A special property of the Kramers-Kronig relationships is of importance for this discussion, the punctual behaviour of the transformation equations. Partial integration of (8a) gives the following relationship

$$
\Delta \varphi(x)=\frac{1}{2 \pi x} \int_{0}^{\infty} \ln \left|\frac{x+\omega}{x-\omega}\right|^{\mathrm{d} \omega \ln \frac{R}{R_{\mathrm{ref}}}} \frac{\mathrm{d} \omega}{\mathrm{d} \omega} .
$$

The function $\ln (x+\omega) /(x-\omega)$, the weighting factor, is shown in figure 2 . It follows from its behaviour that only the in $R / R_{\text {ref }}$ values in the immediate vicinity of $x$ are of importance to the integral. Thus the tailfit in most cases is only of small influence to the 


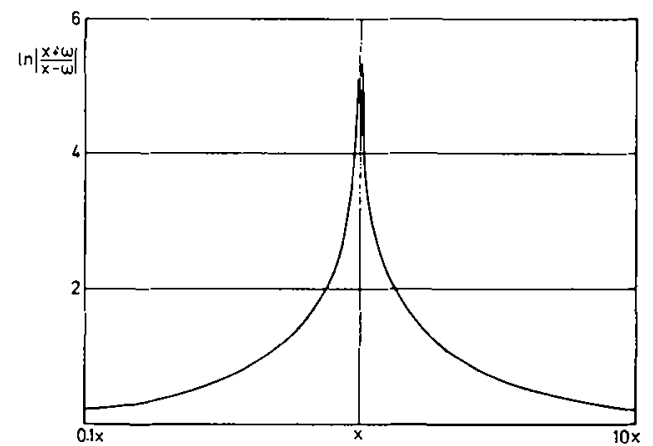

Fig. 2. - The factor $\ln (\omega+x) /(\omega-x)$ against $\omega$ in eq. (10)

$\Delta \varphi$-values. There are also experimental methods for testing the tailfit procedure (compare chapter 6). The integral is now separated into two parts for the numerical calculation of the principal value. The integration limits of the two parts are $0 \rightarrow \omega=x-\delta$ and $\omega=x+\delta \rightarrow \infty$. $\delta$ is varied until the value of the integral is constant within the desired accuracy. In some cases a simplified summation formula of DeVries et al. [5] could be used.

5. Determination of the anisotropy of the optical constants of platinum oxide. - Experimental results were first obtained by Naegele and Plieth [6] for platinum oxide. In figure 3 the measured $\Delta R / R$-values for

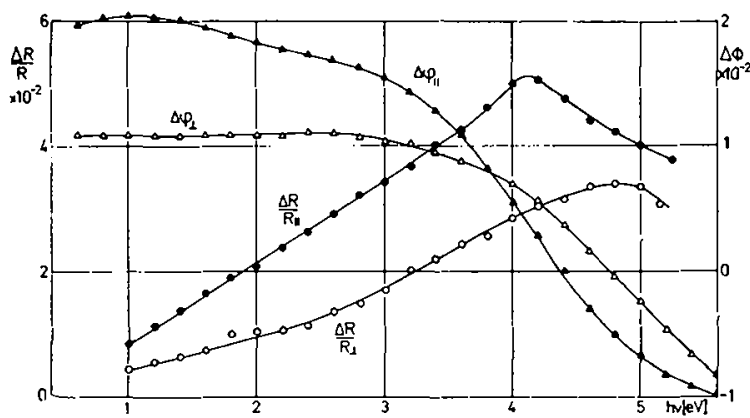

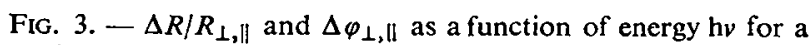
platinum electrode in $0.01 \underline{\mathrm{N}} \mathrm{H}_{2} \mathrm{SO}_{4} / 1 \mathrm{~N} \mathrm{Na}_{2} \mathrm{SO}_{4}$ when the potential is switched between $1.4 \mathrm{~V}$ and $0.5 \mathrm{~V}$ against a NHE, $\alpha_{1}=45^{\circ}$ (Naegele and Plieth [6]).

perpendicular and parralel polarization of a platinum oxide are shown, when switching the potential between $0.5 \mathrm{~V}$ and $1.4 \mathrm{~V}$ against NHE. For the tailfit the curves were fitted by a polymonial and extrapolated for $h v>5.2 \mathrm{eV}$ until $\Delta R / R=0$ and for $h v<0.9 \mathrm{eV}$ to $h v=0 \mathrm{eV}$. The results of these phase calculations are also shown in figure 3 . In the next step integrals

$$
\int_{1}^{3}\left(\hat{\varepsilon}_{\mathrm{t}}-\hat{\varepsilon}_{\mathrm{ref}}\right) \mathrm{d} z \text { and } \int_{1}^{3}\left(\frac{1}{\hat{\varepsilon}_{\mathrm{n}}}-\frac{1}{\hat{\varepsilon}_{\mathrm{ref}}}\right) \mathrm{d} z
$$

were calculated. The results are shown in figures 4 and 5. So far all calculation could be made without any special model about the phase boundary.

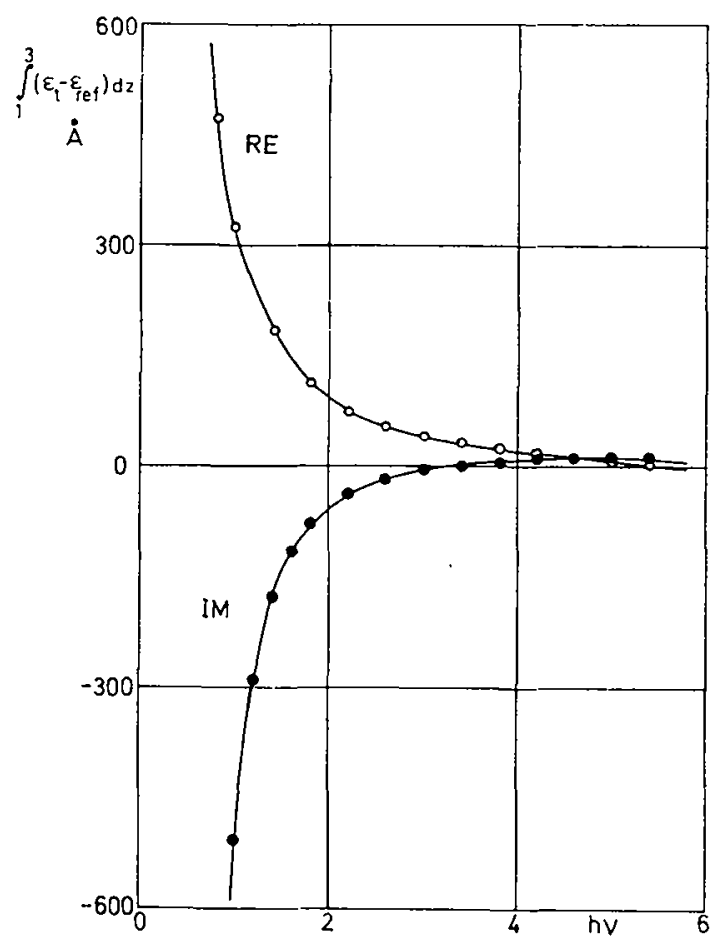

FIG. 4. - Real and imaginary part of the $\int\left(\hat{\varepsilon}_{\mathrm{t}}-\widehat{\varepsilon}_{\mathrm{ref}}\right) \mathrm{d} z$ as function of the photon energy, calculated from the $\Delta R_{\perp}$ and $\Delta \varphi_{\perp}$ values in figure 3 (Plieth and Nacgele [1]).

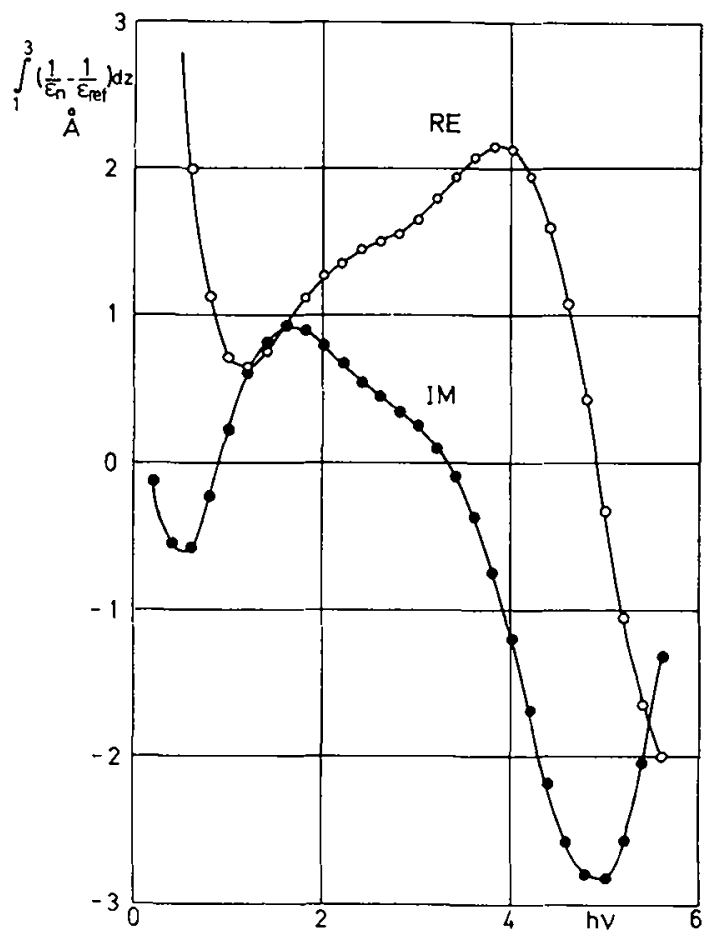

FIG. 5. - Real and imaginary part of the $\int\left(1 / \widehat{\varepsilon}_{\mathrm{n}}-1 / \widehat{\varepsilon}_{\text {res }}\right) \mathrm{d} z$ as function of the photon energy, calculated from the $\Delta R_{\|}$and $\Delta \varphi_{\|}$values in figure 3 (Plieth and Naegelc [1]).

The values of the integrals in figures 4 and 5 are very different in their magnitude. The integral for the tangential component is of the order of $100 \AA$ while the 
TABLE I

$$
\widehat{\varepsilon}_{\text {ref }}
$$

Weak absorbing

$$
\varepsilon_{\mathrm{rcf}}^{\prime} \geqslant 1, \varepsilon_{\mathrm{rcf}}^{\prime \prime} \ll 1
$$

$$
\Delta \varepsilon^{\prime} \gtrless 0
$$

$$
\Delta \varepsilon^{\prime} \gtrless 0
$$

Moderate absorbing

$$
\varepsilon_{\mathrm{ref}}^{\prime}>0,0,1<\varepsilon_{\mathrm{rcf}}^{\prime \prime}<1
$$

$\Delta \varepsilon^{\prime \prime}<0$

Absorbing

$$
\Delta \varepsilon^{\prime \prime} \gtrless 0,\left|\Delta \varepsilon^{\prime \prime}\right| \ll 1
$$$$
\Delta\left(\frac{1}{\widehat{\varepsilon}}\right)^{\prime} \gtrless 0
$$$$
\varepsilon^{\prime} \geqslant 1
$$$$
-1<\Delta\left(\frac{1}{\hat{\varepsilon}}\right)<+1
$$$$
\Delta\left(\frac{1}{\hat{\varepsilon}}\right)^{\prime \prime} \gtrless 0,\left|\Delta\left(\frac{1}{\frac{\hat{\varepsilon}}{\varepsilon}}\right)^{\prime \prime}\right| \ll 1
$$$$
\Delta\left(\frac{1}{\hat{\varepsilon}}\right)^{\prime \prime}<0
$$

$\varepsilon^{\prime \prime} \ll 1$

$$
\Delta \varepsilon^{\prime} \gtrless 0
$$

$\Delta \varepsilon^{\prime} \gtrless 0$

Absorbing

$$
0<\Delta \varepsilon^{\prime \prime}<1
$$$$
-1<\Delta \varepsilon^{\prime \prime}<+1
$$

$\varepsilon^{\prime}>0$

$$
\Delta\left(\frac{1}{\hat{\varepsilon}}\right)^{\prime} \gtrless 0
$$

$0,1<\varepsilon^{\prime \prime}<1 \quad \Delta\left(\frac{1}{\widehat{\varepsilon}}\right)^{\prime \prime}>0$

Strong

Absorbing

$$
\Delta \varepsilon^{\prime}<0,\left|\Delta \varepsilon^{\prime}\right|>1
$$$$
\Delta \varepsilon^{\prime \prime} \gg 1, \quad \Delta \varepsilon^{\prime \prime} \simeq \varepsilon^{\prime \prime}
$$

$\varepsilon^{\prime}<0$

$$
\Delta\left(\frac{1}{\widehat{\varepsilon}}\right)^{\prime}<0
$$

$$
\Delta\left(\frac{1}{\frac{\lambda}{\varepsilon}}\right)^{\prime \prime} \gtrless 0,\left|\Delta\left(\frac{1}{\hat{\varepsilon}}\right)^{\prime \prime}\right| \ll 1 \quad \Delta\left(\frac{1}{\hat{\varepsilon}}\right)^{\prime \prime}<0
$$$$
\Delta\left(\frac{1}{\hat{\varepsilon}}\right)^{\prime} \gtrless 0
$$$$
\Delta\left(\frac{1}{\hat{\varepsilon}}\right)^{\prime \prime} \gtrless 0
$$

$\Delta \varepsilon^{\prime}<0$

$\Delta \varepsilon^{\prime \prime} \gg 1$

$\varepsilon^{\prime \prime} \gg 1$

\section{Strong absorbing}

$\varepsilon_{\mathrm{ref}}^{\prime}<0, \varepsilon_{\mathrm{ref}}^{\prime \prime} \gg 1$

$\Delta \varepsilon^{\prime}>0$

$\Delta \varepsilon^{\prime \prime}<0,\left|\Delta \varepsilon^{\prime \prime}\right| \simeq \varepsilon_{\mathrm{rcf}}^{\prime \prime} \gg 1$

$\Delta\left(\frac{1}{\hat{\varepsilon}}\right)^{\prime}>0$

$\Delta\left(\frac{1}{\hat{\varepsilon}}\right)^{\prime \prime} \gtrless 0,\left|\Delta\left(\frac{1}{\hat{\varepsilon}}\right)^{\prime \prime}\right| \ll 1$

$\Delta \varepsilon^{\prime}>0$

$\Delta \varepsilon^{\prime \prime}<0,\left|\Delta \varepsilon^{\prime \prime}\right| \gg 1$

$\Delta\left(\frac{1}{\widehat{\varepsilon}}\right)^{\prime}>0$

$\Delta\left(\frac{1}{\hat{\varepsilon}}\right)^{\prime \prime}>0$

$\Delta \varepsilon^{\prime} \gtrless 0$

$\Delta \varepsilon^{\prime \prime} \gtrless 0$

$\Delta\left(\frac{1}{\hat{\varepsilon}}\right)^{\prime} \gtrless 0,\left|\Delta\left(\frac{1}{\hat{\varepsilon}}\right)^{\prime}\right| \ll 1$

$\Delta\left(\frac{1}{\hat{\varepsilon}}\right)^{\prime \prime} \gtrless 0,\left|\Delta\left(\frac{1}{\hat{\varepsilon}}\right)^{\prime \prime}\right| \ll 1$ integral for the normal component lies between +3 and $-3 \AA$. At mean angles of incidence the coefficients relating the integral values and $\Delta R / R$ and $\Delta \varphi$ are not too different in magnitude. Thus at these angles of incidence $\Delta R / R_{\|}$and $\Delta \varphi_{\|}$are mainly determined by the integral for the tangential component. Otherwise the experimental accuracy in $\Delta R / R_{\|}$and hence in $\Delta \varphi_{\|}$is influencing the normal component much stronger than the tangential component. Especially for photon energies below $2 \mathrm{eV}$ this effect becomes predominating.

To get an impression of the values of the integrals various experimental situations are discussed in table $I$. The values of $\widehat{\varepsilon}$ and $\widehat{\varepsilon}_{\text {ref }}$ have been differentiated for weak, intermediate and strong absorption. Multiplying the $\Delta \hat{\varepsilon}$ and $\Delta \mathrm{l} / \hat{\varepsilon}$ values by an appropriate layer thickness (e. g. $10 \AA$ ) the integral values to be expected are obtained. Comparing the results for platinum/platinum oxide with this table they agree at low photon energies $(<1 \mathrm{eV})$ with the values expected for a weak absorbing film and a strong absorbing reference state. For higher photon energies $(>2 \mathrm{eV}$ ) they agree better with the values of a strong absorbing film and a strong absorbing reference state.

Values of $\widehat{\varepsilon}_{\mathrm{t}}$ and $\widehat{\varepsilon}_{\mathrm{n}}$ were calculated from the integrals. For the calculation $\hat{\varepsilon}_{\text {ref }}$ was set $\hat{\varepsilon}_{3}$. This is equal to a model with the oxide layer growing into the platinum metal $\left({ }^{1}\right)$. The thickness $d$ was varied. Mean values of $\hat{\varepsilon_{t}}$ and $1 / \hat{\varepsilon}_{\mathrm{n}}$ were determined by this procedure. From these values $n_{t}, k_{t}, n_{n}$ and $k_{n}$ were derived. Results for $9 \AA$ are shown in figure 6 . Especially for the $k_{\mathrm{t}}$ - and $k_{\mathrm{n}}$ - values a preliminary interpretation was given in the original paper [6].

The calculated optical constants depended strongly on the d-values as well as on the set of optical constants for the metal. They certainly also depend on the model

(1) It is also in agreement with a ratio $\widehat{r} / \hat{r}_{\text {res }}$ without phase correction as obtained with the Kramers/Kronig transformation. A model where the oxide layer grows also into the electrolyte phase would imply for the reference value $\hat{\varepsilon}_{\text {ret }}=\hat{\varepsilon}_{1}$ along a distance $d_{1}$ and $\widehat{\varepsilon_{3}}$ along a distance $d_{3}$. This would also imply an additional phase correction (compare chapter 7 ). 


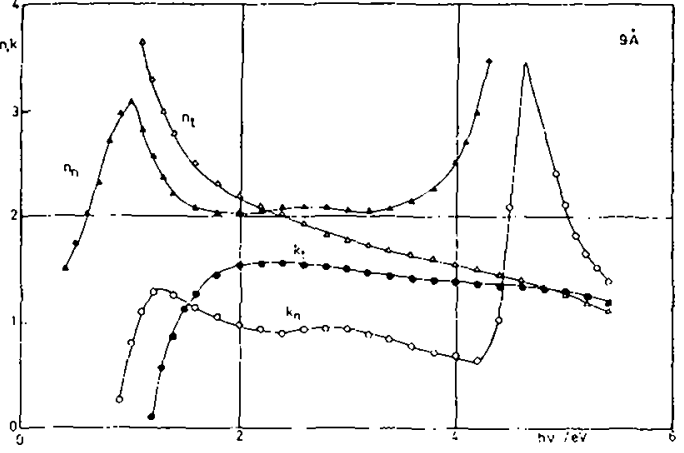

Fig. 6. - Tangential and normal component of the refraction indices and absorption coefficients $n_{\mathrm{t}}, n_{\mathrm{n}}, k_{\mathrm{t}}$ and $k_{\mathrm{n}}$ as function of the photon energy, calculated from the integrals in figure 4 and 5 with $\widehat{\varepsilon}_{\mathrm{re}}=\widehat{\varepsilon}_{3}$ and a surface layer thickness of $9 \AA$ (Naegele and Plieth [6]).

for the interface and the assumed reference value $\hat{\varepsilon}_{\text {ref }}$. In the values $n_{n}$ and $k_{n}$ one should also consider the higher sensitivity to the experimental accuracy. Thus the physical significance of the data in figure 6 is still open to further discussion. It is probably more promising to discuss the change in the optical constants, given by the integrals (11), than to try calculation of absolute values.

6. Determination of the orientation of adsorbed organic molecules. - When organic molecules are adsorbed on an electrode surface the change in the optical properties of the surface is much smaller than for oxide formation.

In many cases a reference value, a film frec surface, cannot be obtained by potential variation. Even if a desorption potential exists the electromodulation effect dominates the optical variations [7]. To investigate such layers we have to look for optical properties specific for the adsorbed molecules. The electrochromic effect is such a property.

The light absorption is influenced by an electric field. For free atoms this effect is known as the Stark effect. For molecules in dense media the origins for the absorption changes are of more complex nature and are called Electrochromism. By modulating the field across the double layer by potential modulation field induced changes of light absorption can also be measured for molecules within the electrolytic double layer. The effect was first detected for Rhodamin B adsorbed on platinum and silver by Gruschinske and Plieth [8].

In figure 7 the spectrum of the modulated reflectivity of a platinum electrode in $0.1 \mathrm{~N} \mathrm{H}_{2} \mathrm{SO}_{4} / 0.9 \mathrm{~N} \mathrm{Na}_{2} \mathrm{SO}_{4}$ is shown (curve a). In curve $b$ Rhodamin $B$ was added to the electrolyte. Figure $8 a$ shows the difference between similar measurements $\left({ }^{2}\right)$ for parallel and perpendicular polarized light. Figure $9 a$ shows analogue experiments on a silver electrode.

If one compares the curves in figures $8 a$ and figure $9 a$ with the spectrum of Rhodamin B and the derivatives

(2) Only the deviations from the base line are shown in figure 8 .

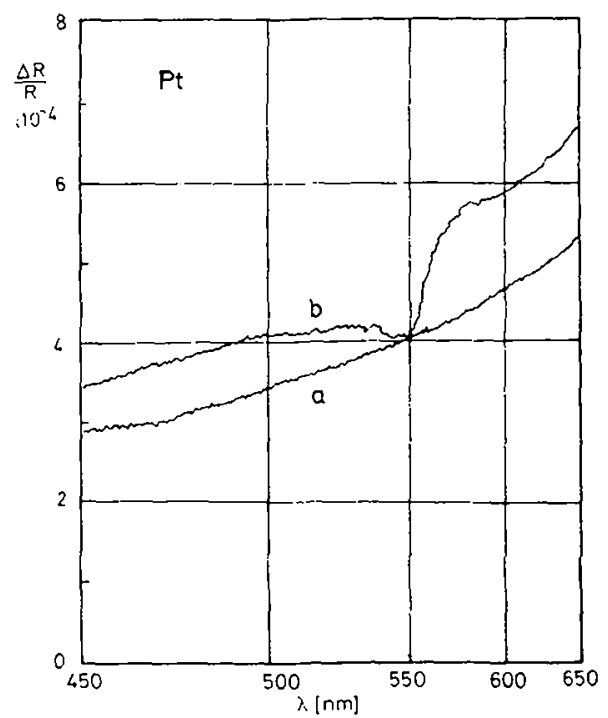

FiG. 7. - Modulated reflectivity $\Delta R / R$ of a platinum electrode in $0.1 \mathrm{~N} \mathrm{H}_{2} \mathrm{SO}_{4} / 1.0 \mathrm{~N} \mathrm{Na} \mathrm{SO}_{4}$ (curve a) and with addition of $10^{-5} \mathrm{M}$ rhodamin $\bar{B}$ (curve b), $E_{0}=600 \mathrm{mV}$ against $\mathrm{SCE}$, $\Delta E= \pm 100 \mathrm{mV}, \alpha_{1}=65^{\circ}$, ! polarization (Gruschinske and Plieth [8]).

of this spectrum [9] the curves $8 a$ and $9 a$ are very like the first derivative of the Rhodamin spectrum. Considering also the dependence on the electrode potential $E_{0}$ and on the modulation amplitude $\Delta E$ the effect can be described as the change of the absorption coefficient of Rhodamin $B$ with the field modulation $\Delta F \sim \Delta E$

$$
\Delta k=A \Delta E+B E_{0} \Delta E .
$$

The first term $A \Delta E$ is the more important term. It follows from the theory of electrochromism that this term is proportional to the difference of the dipole moment in the excited and in the ground state.

The Rhodamin $B$ molecule has the following formula

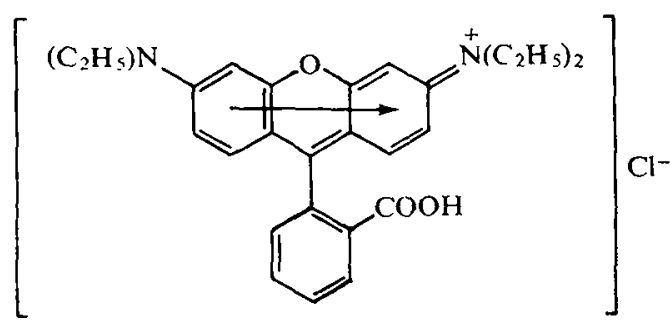

The transition moment for the investigated absorption bond lies in the conjugated $\pi$-system indicated by the arrow. The dipole moment is determined by the carboxylic groups in the isolated aromatic ring. This ring is twisted to the conjugated system. Thus from the knowledge about the optical properties of the molecule it should be possible to determine its orientation in the surface area if the anisotropy of the $\Delta k$-modulation is determined.

Figures $8 b$ and $9 b$ show the $\Delta \varphi$-calculations. Then the integrals were calculated now representing the variation of the dielectric constants $\Delta \hat{\varepsilon}_{t}$ and $\Delta\left(1 / \hat{\varepsilon}_{n}\right)$ 


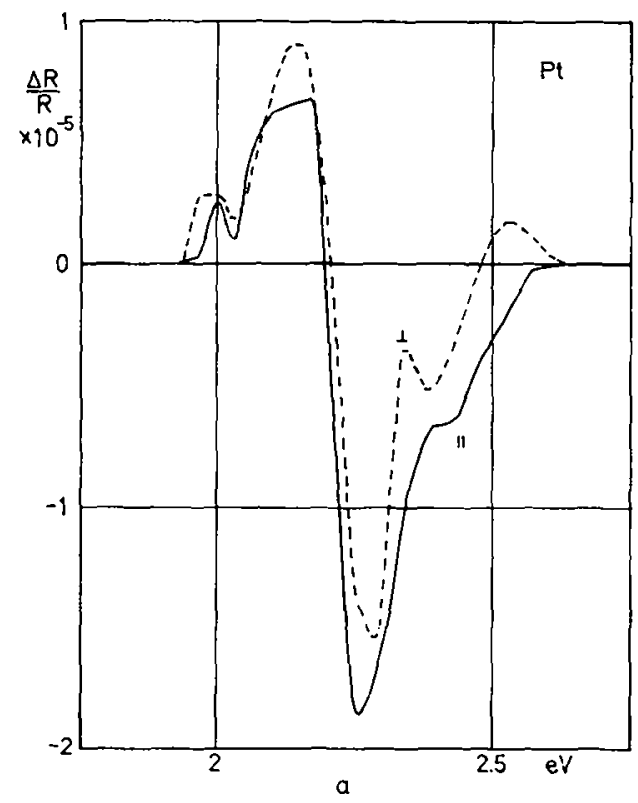

FIG. 8a. - Modulated reflectivity $\Delta R / R$ of a platinium electrode in $10^{-5} \mathrm{M}$ rhodamin $B / 0.1 \mathrm{~N} \mathrm{H}_{2} \mathrm{SO}_{4} / 1.0 \mathrm{~N} \mathrm{Na} \mathrm{SO}_{4}$ after background substraction, $E_{0}=100 \mathrm{mV}$ against $\overline{S C E}, \Delta E= \pm 50 \mathrm{mV}$, 1 , \| polarization, $\alpha_{1}=65^{\circ}$.

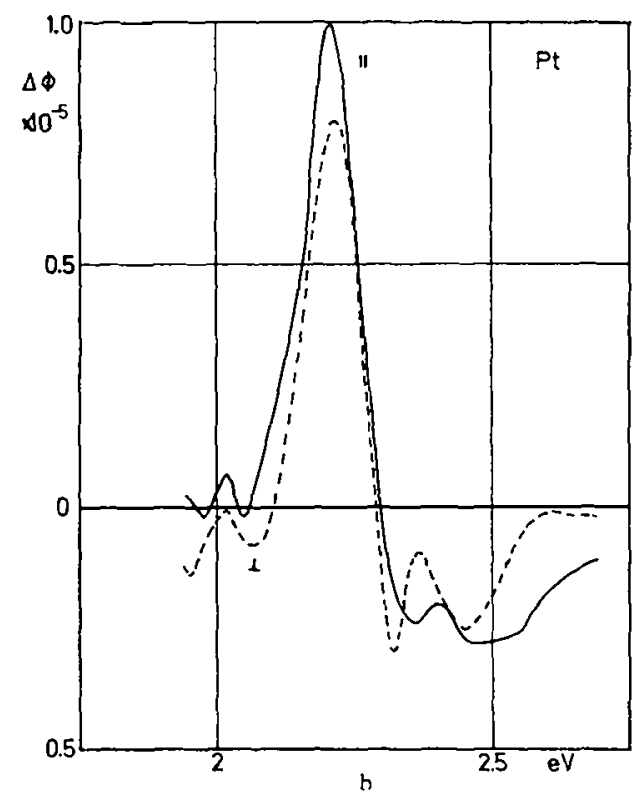

FIG. $8 b .-\Delta \varphi$-values obtained from $a$ ) by Kramers-Kronig transformation (Plieth, Hensel, Gruschinske, to be published).

of the adsorption layer with the potential modulation $\Delta E$. The results are shown in figure 10 for platinum and in figure 11 for silver.

From the spectroscopic values of Rhodamin B one would expect moderate absorption for a monomolecular film. This was confirmed by Reich et al. [9] for adsorption layers of Rhodamin $B$ on fatty acids. From table $I$ follows that for such films values of similar magnitude are expected for $\Delta \widehat{\varepsilon}$ and $\Delta(1 / \hat{\varepsilon})$. Comparing with the results in figures 10 and 11 the values for $\mathrm{Pt}$ as well

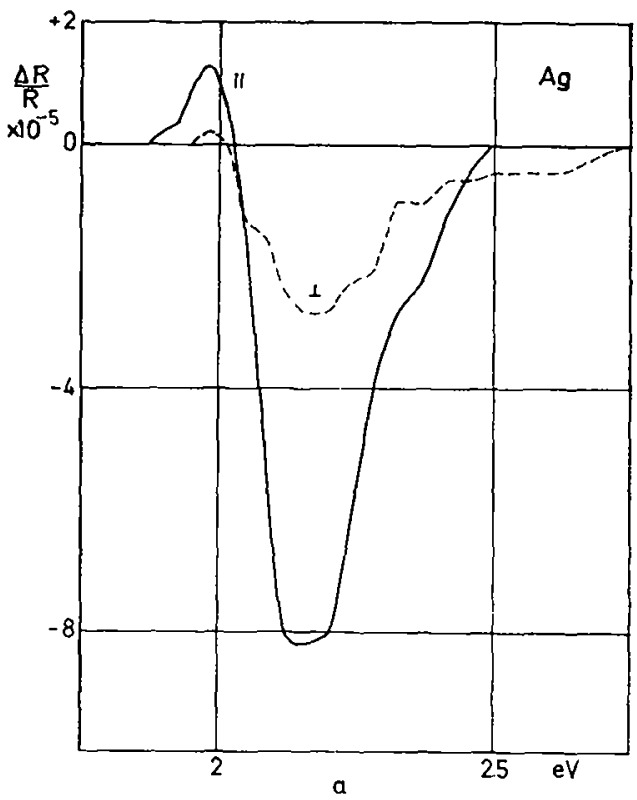

FIG. 9a. - Modulated reflectivity $\Delta R / R$ of a silver electrode in $10^{-5} \mathrm{M}$ rhodamin $B / 0.1 \mathrm{~N} \mathrm{~N}_{2} \mathrm{SO}_{4} / 1.0 \mathrm{~N} \mathrm{Na}_{2} \mathrm{SO}_{4}$ after background substraction, $E_{0}=\overline{\mathrm{OV}}$ against $\mathrm{SCE}, \Delta E= \pm .10 \mathrm{mV}$, $\perp, / /$ polarization, $\alpha_{1}=65^{\circ}$.

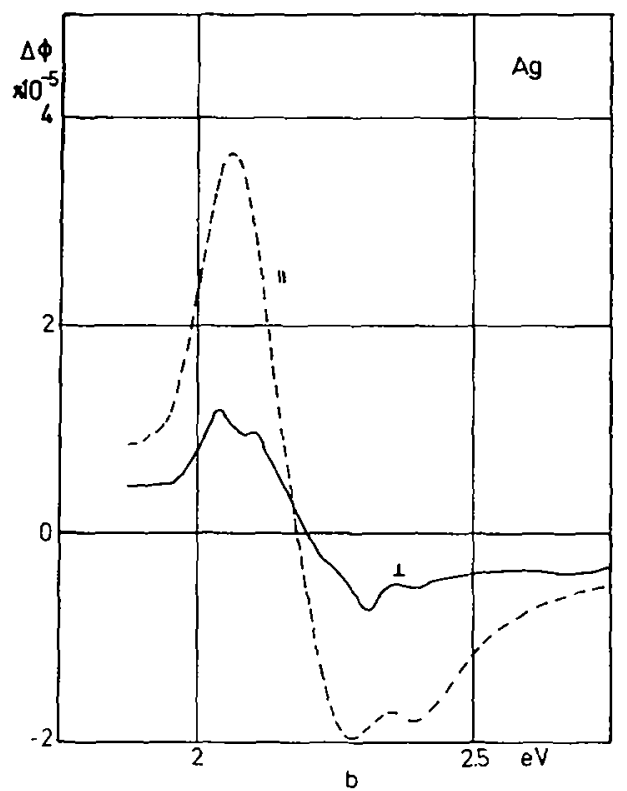

FiG. $9 b .-\Delta \varphi$-values obtained from $a$ ) by Kramers-Kronig transformation (Plieth, Hensel, Gruschinske, to be published).

as for $\mathrm{Ag}$ differ by two orders of magnitude : $\Delta \hat{\varepsilon}_{\mathrm{t}} \gg \Delta\left(1 / \widehat{\varepsilon}_{\mathrm{n}}\right)$. Furthermore the absolute values of $(1 / \hat{\varepsilon})^{\prime}$ and $(1 / \hat{\varepsilon})^{\prime \prime}$ are smaller by a factor $10^{-2}$ to $10^{-3}$ than expected from table $\mathrm{I}$. This behaviour is explained by a tangential orientation of the conjugated $\pi$-system and the transition moment on the surface. Most likely the tangential orientation is identical with a fiat orientation of the large $\pi$-system on the electrodes. These results are identical for evaporated $\mathrm{Ag}$ as well as for mechanically polished Pt. Otherwise the difference for 

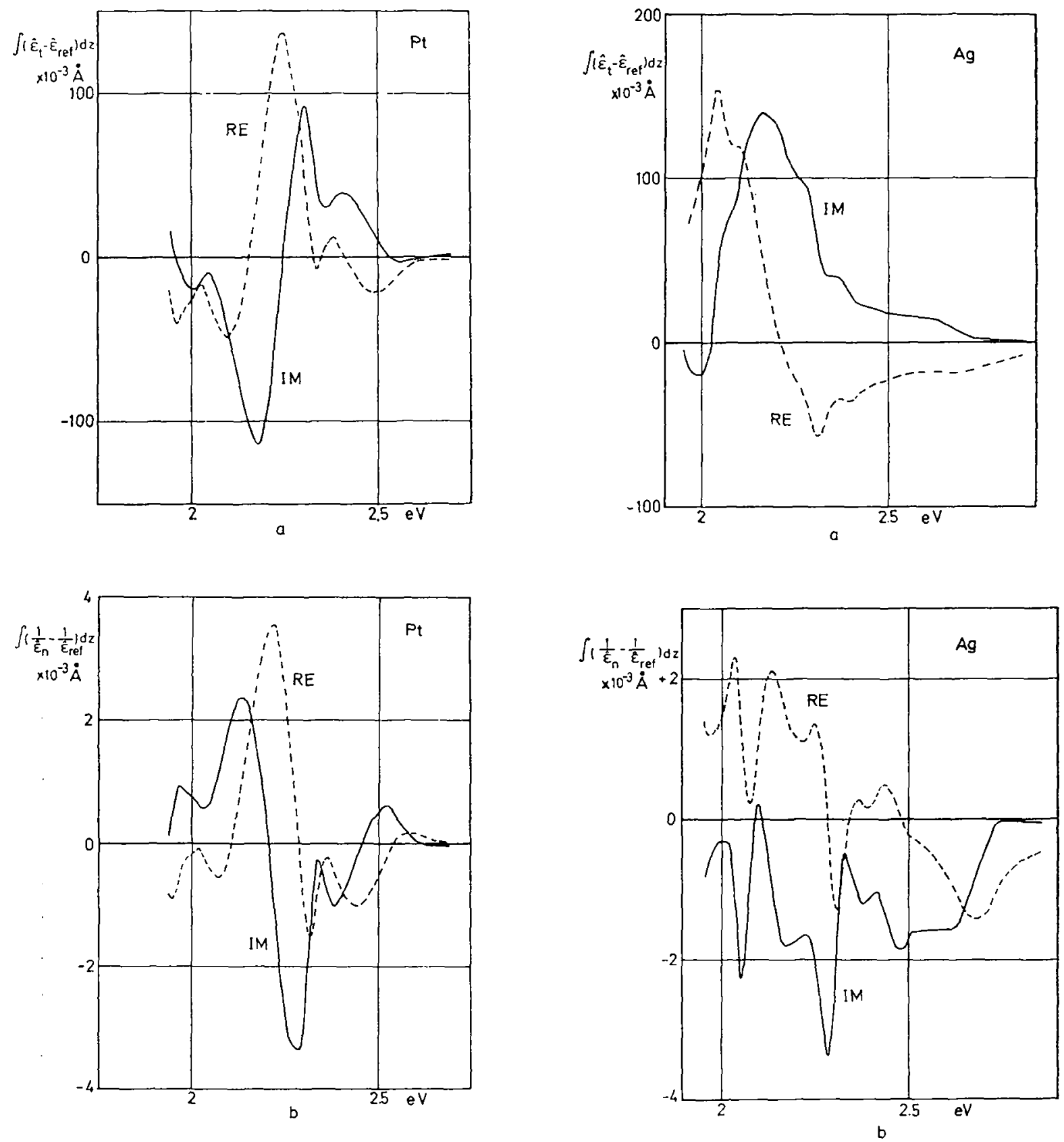

FrG. 10. - Real an imaginary parts of $\int\left(\hat{\varepsilon}_{\mathrm{t}}-\widehat{\varepsilon}_{\mathrm{ret}}\right) \mathrm{d} z(a)$ and of $\int\left(1 / \hat{\varepsilon}_{\mathrm{n}}-1 / \hat{\varepsilon}_{\mathrm{ret}}\right) \mathrm{d} z(b)$ for rhodamin $B$ adsorbed on platinium, calculated from the data in figure 8, (Plieth, Hensel, Gruschinske. to be published).

platinum is clearly smaller than for Ag. This can be explained by surface roughness.

7. Experimental determination of the phase shift. The Kramer-Kronig transformation for determining the phase change $\Delta \varphi$ has two disadvantages. It needs measurements over a wide frequency range and it is sensitive to a displacement of the phase boundary. This can be seen best by the following experiment. If one deposits on a metal electrode (reference system)

FIG. 11. - Real and imaginary parts of $\left(\widehat{\varepsilon_{t}}-\widehat{\varepsilon_{\text {ret }}}\right) \mathrm{d} z(a)$ and of $\left(1 \hat{\varepsilon_{\mathrm{n}}}-1 / \hat{\varepsilon}_{\mathrm{ref}}\right) \mathrm{d} z(b)$ for rhodamin $B$ adsorbed on silver, calculated from the data in figure 9 (Plieth, Hensel, Gruschinske, to be published).

a layer of the electrode metal of thickness $d_{1}$ the change in reflectivity is zero and hence the $\Delta \varphi$-value obtained by transformation is zero. Otherwise the phase boundary is shifted by $d_{1}$ and the ratio of the Fresnel reflection coefficients $\hat{r} / \hat{r}_{\text {ref }}$ has the form

$$
\frac{\hat{r}}{\hat{r}_{\mathrm{ref}}}=1+i \frac{4 \pi}{\lambda} d_{1} n_{1} \cos \alpha_{1} .
$$

Thus $\hat{r} / \hat{r}_{\text {ref }}$ obtained by a Kramers-Kronig transfor- 
mation has to be corrected for this boundary shift by multiplying with a phase factor

$$
\frac{\hat{r}}{\hat{r}_{\mathrm{ref}}}=\left.\frac{\hat{r}}{\hat{r}_{\mathrm{ref}}}\right|_{\mathrm{KK}} \cdot \exp \left(i \frac{4 \pi}{\lambda} d_{1} n_{1} \cos \alpha_{1}\right) \text {. }
$$

To overcome these disadvantages we have looked for a method for an experimental determination of the Fresnel reflection coefficient. Measurements of the phase relations of two light beams are possible by interferometry. Unfortunately, the sensitivity of this method in its usual manner is not as high as to allow phase difference measurements of the order of $1 / 1000$ th of a wavelength. But if one could use in interferometry the sensitivity enhancement the modulation technique has brought to reflectance spectroscopy measurements in the $\AA$-region should become possible. This was proposed by Plieth and Bruckner [10] and the apparatus we have built for these experiments is shown in figure 12. The interferometer is of the Michelson type with some variations necessary for measurements with electrochemical systems. Polarized light (in general of a tunable laser source) is splitted into two beams reflected twice on the electrodes in the electrochemical cells EC and additionally on the mirrors MI and is then recombined behind the beam splitter. The magnified interference fringe system is focussed on a photomultiplier. By an aperture in front of the multiplier a special position of the fringe system is selected. If by modulation of the electrode potential in one electrochemical cell $\left(E C_{1}\right)$ the phase shift $\Delta \varphi_{1}$ of this beam is modulated then the fringe system and the intensity in the apertur oscillate. The intensity modulation $\Delta I$ can be measured very sensitive with a lock-in amplifier. Measuring also the interfering intensities $I_{1}$ and $I_{2}$ of the splitted light

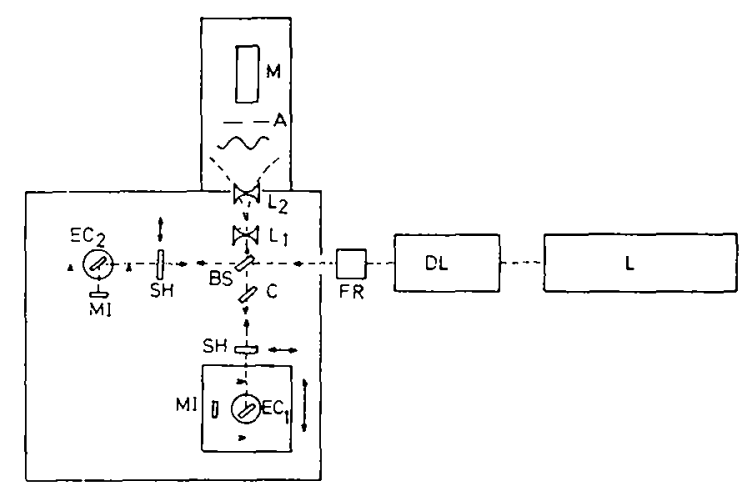

Fig. 12. - Optical set-up for combinated modulated reflectance spectroscopy and modulated interferometry, L argonlaser, DL dye laser, FR Fresnel rhombus, BS beam splitters, $C$ compensator, SH shutter, EC electrochemical cell, MI mirror, $L_{1}, L_{2}$ lenses, A pinhole aperture.

beams and the reflectance modulation $\Delta I_{1}$ of $\mathrm{EC}_{1}$, the phase shift $\Delta \varphi_{1}$ can be calculated.

The interferometric $\Delta \varphi$-value is not only the $\Delta \varphi$ value related to $\Delta R / R$ by a causal transform. It also contains the phase factor of the displacement of the interface during the modulation. By comparison of the interferometric $\Delta \varphi$-values and of calculated $\Delta \varphi$-values this boundary shift can be determined. Thus the experimental determination of $\Delta \varphi$ not only substitutes the calculation method but really extends the possibilities of optical measurements.

This work was supported by the Deutsche Forschungsgemeinschaft and by the Fonds der Chemischen Industrie. We appreciate the financial assistance of these institutions.

\section{References}

[1] Plieth, W., Naegele, K., Surf. Sci. 64 (1977) 484.

[2] Plieth, W., Naegele, K., Surf. Sci. 50 (1975) 53.

[3] HaAs, J., Schmidt, H. L., Edts., Mathematisches Wörterbuch, 3. Auff., Teubner 1967.

[4] LANDaU, L. D., Lipschitz, E. M., Lehrbuch der theoretischen Physik (Akademie-Verlag) 1967, Bd. 8, p. 302.

[5] Emeis, C. A., OOSterhoff, L. Y., De Vries, G., Proc. R. Soc. A 297 (1967) 54.

[6] Naegele, K., Plieth, W., Surf. Sci. 50 (1975) 64 ; 61 (1976) 504.
[7] Plieth, W., Bruckner, H., Z. phys. Chem. (Frankfurt/Main) 98 (1975) 33.

[8] Gruschinske, P., Plieth, W., 27th meeting of ISE, Zürich 1976, Extended Abstracts No. 208 ;

Gruschinske, P., Dissertation, Freie Universität Berlin 1976.

[9] Reich, R., Schmidt, S., Ber. Bunsenges. phys. Chem. 76 (1972) 589.

[10] Plieth, W., Bruckner, H., Surf. Sci. 66 (1977) 357.

[11] Toll, J. S., Phys. Rev. 104 (1856) 1760.

\section{DISCUSSION}

M. Dignam (a Comment). - Your integral approach, Dr Abeles' surface conductivity tensor approach and our surface susceptibility approach are all exactly equivalent. In each, two complex values surface optical constants completely characterize the optical response of the system. I believe it is very important for those working in the field to realize that and take it into account in their analysis of optical data.
W. Plieth. - I completely agree with the comment of Pr. Digman. One should also point out that the concept of molecular oscillators of Strachan and of Shivunin leads to similar equations.

J. P. RANDIN. - I would like to ask further informations concerning the electrochromism of the Rhodamin B on the Pt electrode. 
a) Is the adsorption of the molecule limited to the monolayer?

b) What is the response time and reversibility of the phenomenon?

c) What electrolyte did you use ?

d) Do you have any idea of the absorption (or transmission change induced by the variation of the potential?

W. Plietr. - To your question a) We did not determine the total amount of rhodamin $B$. The concentration dependance of the modulated reflectivity (increasing the concentration of rhodamin B from $10^{-5} \mathrm{M}$ to $10^{-4} \mathrm{M}$ ) indicates the formation of dimers at higher concentrations.

Question $b$ ) The current-potential curve shows no electrochemical processes (oxidation, reduction, reorientation of the adsorbed molecules). The response time of the absorption shift due to the electric field is neglectable for the modulation frequency of $32 \mathrm{~Hz}$.

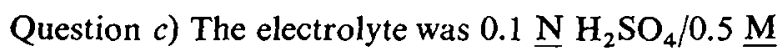
$\mathrm{Na}_{2} \mathrm{SO}_{4}$. We also made measurements in alcaline solution.

Question d) From the integral values I gave in figures 10 and 11 , one calculates a maximum of

and of

$$
\Delta \varepsilon_{\mathrm{t}}^{\prime}=2 k_{\mathrm{t}} \Delta k_{\mathrm{t}}
$$

$$
\Delta \varepsilon_{\mathrm{\imath}}^{\prime \prime}=2 n_{\mathrm{t}} \Delta k_{\mathrm{t}}
$$

of the order of magnitude of 0,01 (modulation amplitude $\pm 50 \mathrm{mV}, \Delta n_{\mathrm{t}} \simeq 0, d=10 \AA$ ). Thus one can estimate: $\Delta k_{t} c a 0.1$ per volt $(10 \%)$.

W. PaAtsch. - Using the Fresnel coefficients developed by you it was pointed out that one does not need the thickness of a surface layer as it is integrated bet- ween phase 1 and phase 3 . In the calculation of $n$ and $\mathrm{k}$ for platinum oxide, however, you had to assume a definite thickness value. Could you please explain this ?

W. Plietr. - In the framework of the Maxwell theory assuming uniaxial anisotropy, one can calculate only the integrals I have shown. They have the dimension of a length. When you like to calculate other quantities like $n$ and $k$, you have to introduce assumptions.

The first assumption regards the thickness of the distortion in the optical properties at the interface. When you introduce a thickness you calculate mean differences.

$$
\begin{aligned}
& \hat{\varepsilon}_{\mathrm{t}}-\widehat{\varepsilon}_{\mathrm{ref}}=\frac{1}{d} \int_{1}^{3}\left(\widehat{\varepsilon_{\mathrm{t}}}-\widehat{\varepsilon}_{\mathrm{ref}}\right) \mathrm{d} z \\
& \frac{1}{\hat{\varepsilon}_{\mathrm{n}}}-\frac{1}{\hat{\varepsilon}_{\mathrm{ref}}}=\frac{1}{d} \int_{1}^{3}\left(\frac{1}{\hat{\varepsilon}_{\mathrm{n}}}-\frac{1}{\hat{\varepsilon}_{\mathrm{ref}}}\right) \mathrm{d} z .
\end{aligned}
$$

The magnetude of these differences was discussed in the table I have shown. The second assumption regards the reference value.

You can assume that this value is equal to $\widehat{\varepsilon}_{3}$. That means the layer is formed entirely in phase 3 . This is the Mc Intyre-Aspnes model. You can assume that this value is $\varepsilon_{1}$. Then the layer is formed in phase 1 , or you can use some more complicated mixture of both. The value you then calculate is $\hat{\varepsilon}_{\mathrm{t}}$ and $1 / \widehat{\varepsilon}_{\mathrm{n}}$.

Writing the last value simply as $\widehat{\varepsilon}_{\mathrm{n}}$ means that you assume furthermore a constant region of $\hat{\varepsilon}_{n}$.

It was one of my intentions just to show how many assumptions are necessary for obtaining these data and I proposed that in many cases it might be more fruitful to discuss the integrals and not to calculate absolute optical constants. 